Original Research

\title{
Experimental Phenomenology as Personalized Health Intervention: A Case Illustration
}

Lars-Gunnar Lundh *

Department of Psychology, Lund University, Box 213, 22100 Lund, Sweden; E-Mail: LarsGunnar.Lundh@psy.lu.se

* Correspondence: Lars-Gunnar Lundh; E-Mail: Lars-Gunnar.Lundh@psy.lu.se

Academic Editor: Peta Stapleton

Special Issue: Mind-Body Approaches that are Revolutionizing the Health Field

OBM Integrative and Complementary Medicine Received: July 27, 2021

2022, volume 7 , issue 1

Accepted: January 30, 2022

doi:10.21926/obm.icm.2201008

Published: February 15, 2022

\begin{abstract}
Experimental phenomenology is the investigation of phenomenological practices and their effects. To engage in a phenomenological practice means to focus attention on aspects of one's experiences (e.g., sense impressions or body sensations) while adopting a specific kind of attitude (e.g., an explorative attitude) according to some set of instructions. The basic assumption is that the regulation of attention and attitudes involved in such practices may have effects on people's health and quality of life. The present paper describes the basic principles of experimental phenomenology as an approach to personalized health interventions and illustrates these principles with a case, where a mindful embodiment practice was constructed for an individual suffering from insomnia due to early morning awakenings. The results are discussed in terms of the importance of personalization, the ambition to formulate general conclusions about the effects of phenomenological practices, and the need for measurements not only before and after treatment but also during the treatment process.
\end{abstract}

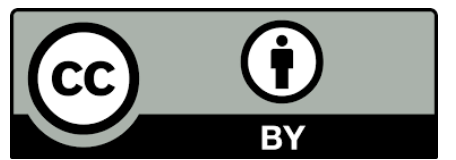

(C) 2022 by the author. This is an open access article distributed under the conditions of the Creative Commons by Attribution License, which permits unrestricted use, distribution, and reproduction in any medium or format, provided the original work is correctly cited. 


\section{Keywords}

Mindfulness; experimental phenomenology; insomnia; personalization; personalized health intervention

\section{Introduction}

The purpose of this study is to present experimental phenomenology as an approach to personalized health interventions. This is done by first describing the basic principles of experimental phenomenology, and then illustrating it with a case description where it was used to construct a personalized health intervention for an individual suffering from insomnia due to early morning awakenings.

\subsection{Experimental Phenomenology as the Study of Phenomenological Practices}

Experimental phenomenology as defined here [1, 2] is the investigation of phenomenological practices and their effects on experiences. Although it has roots in Husserl's [3] philosophical phenomenology and Ihde's [4,5] experimental phenomenology, it does not follow Husserl's or Ihde's philosophy in any orthodox manner. Common to all varieties of phenomenology is a focus on experience. Experimental phenomenology is a subvariety of phenomenology which is characterized by its focus on intentional variations of experience to study the effects of these. In other words, experimental phenomenology differs from theoretical and descriptive varieties of phenomenology as it involves investigating phenomenological practices and their impact on subsequent experience.

Phenomenological practices involve intentional variations of experiencing by means of changes in the direction of attention and the choice of attitude, typically as guided by verbal instructions or self-instructions. Phenomenology in this sense, including experimental phenomenology, is not some kind of "first-person science" but "a collective and intersubjective project" ([6], p. 46-47). It is a scientific endeavor where "potential effects described by one person can be subjected to replication both by the same person and by other persons. Also, conclusions drawn on the basis of this kind of study are hypothetical and provisional and may be modified or specified on the basis of further study" ([1], p. 493).

To engage in a phenomenological practice is to focus attention on some aspects of one's experiences (e.g., sense impressions or body sensations) while adopting a specific kind of attitude (e.g., an explorative attitude) according to some set of instructions. These instructions, in addition to prescribing the focus of attention, describe how a particular attitude is to be expressed. This means that each phenomenological practice can be specified in terms of attitude and focus of attention. Importantly, although the engagement in a phenomenological practice involves a modification of attention and attitude, it need not involve any modification of overt behavior. Basic to experimental phenomenology, however, is the notion of instructions and self-instructions, because these are what define each particular phenomenological practice in detail. Here, the choice of words is all-important as different words carry different meanings.

One example of a phenomenological practice is mindfulness meditation. Several definitions of mindfulness exist in the literature; however, the majority of them mention at least two basic 
elements: (1) to deliberately focus attention on some aspects of the present experience; and (2) to do this with a particular kind of attitude, variously characterized in terms of being accepting and non-judging; kind, friendly, and caring; and exhibiting openness, curiosity, and non-reactivity to experience (e.g., [7, 8]). An important aspect of this is to gently bring the attention back to the present moment when getting distracted. All these instructions are about how one relates to one's inner experiencing and do not have to involve any publicly observable behavior.

Some phenomenological practices do involve observable behaviors. However, if the instructions are purely behavioral, it is not a phenomenological practice. Take breathing exercises as an example. A breathing exercise that simply involves attending to one's breathing just as it is, with no instruction to change it in any manner, is a pure phenomenological practice. However, if the individual is instructed to breathe in a particular way (for example, slowly or deeply) and simultaneously pay close attention to the breathing, this includes a change in the overt behavior and is therefore not a pure phenomenological exercise. However, it is still a phenomenological practice because it involves instructions about the direction of attention. In contrast, if the instructions are only about breathing in a particular manner and do not mention anything about attention or attitudes, it is not a phenomenological practice but a pure behavioral exercise.

What makes experimental phenomenology into a scientific endeavor is the intersubjective nature of the study of phenomenological practices: potential effects described by one person can be subjected to replication both by the same person and by other persons. Also, conclusions drawn from the results of this kind of research are hypothetical and provisional and are likely to be modified or specified in more detail as the results of further study.

\subsection{Importance of Context and Person}

Basic assumptions in experimental phenomenology are that the effects of phenomenological practices are context-and person-sensitive [1]. This means that it is not likely that a particular practice will always be followed by the same type of consequences. An important question is under which conditions certain observed effects appear, and under which conditions they do not (e.g., [9]).

One aspect of this is personalization: what works for one specific person need not work equally well for another. Experimental phenomenology is primarily an idiographic, person-oriented form of research $[10,11]$ focusing on individual persons, where a first purpose is to draw conclusions at the level of the individual. This, however, does not preclude the search for regularities that generalize across individual persons and contexts. On the contrary, experimental phenomenology aims to search precisely for such general principles. Person-oriented research, however, always starts from the individual and subsequently attempts to generalize from individual cases to larger groups of people and contexts. In this manner, it differs from variable-oriented research $[10,11]$ which starts at the group level and conducts statistical analyses of differences between groups (e.g., experimental and control groups) and correlations between different variables. The preference for person-oriented research does not imply that we should give up attempts to formulate general, nomothetic conclusions about what works for people in general; such conclusions, however, should rely on comparisons between different cases of what has worked for specific persons, and not on statistical averages at the group level. 


\subsection{Practices, not Procedures}

Another basic assumption in experimental phenomenology is that phenomenological practices cannot be reduced to procedures or routines [1]. This means that the instructions for a phenomenological practice should not be given at a too detailed level. More specifically, attention is not to be controlled in detail according to some highly specified procedure, of the kind that would be considered "mindless" by Langer [9]. Instead, attention should be relatively freely applied within the here-and-now. What is required, in other words, is not some kind of rigid routine, but an exercise with a given frame and a certain degree of freedom within this frame.

This was illustrated in a previous study [1] by the construction of a personalized mindfulness practice of mindful driving for an individual who had difficulties staying alert during driving. This mindful driving practice consisted of four phases, each characterized by a specific kind of focus: road, traffic, landscape, and sitting. The ability to regulate attention and shift in a smooth manner between these four phases was referred to as "meta-mindfulness attention," or in Langer's [9] terms as "second-order mindfulness," defined as "choosing what to be mindful about" (p. 197).

To summarize, experimental phenomenology entails the exploration and systematic variation of phenomenological practices. In a health context, this may be used (1) to develop personalized practices designed to fit the individual person and (2) to establish the effects of such practices first at an individual level, and then possibly at a more generalized level.

\subsection{Phenomenological Practices in the Treatment of Insomnia}

Insomnia is a common problem with a multi-faceted background. Two kinds of processes contributing to insomnia are (1) various forms of activation or arousal (cognitive, behavioral, emotional, and physiological) that interfere with the deactivation required to fall asleep (e.g., [12, 13]) and (2) dysfunctional beliefs and attitudes about sleep (e.g., beliefs that sleep can be achieved by willful efforts; e.g., [14]). In this context, it has been suggested that the practice of mindfulness meditation may contribute both to improved skills of cognitive deactivation, and to the development of more functional beliefs about sleep, with less focus on willful efforts $[13,15,16]$.

\section{Case Illustration}

The following case describes a personalized intervention using experimental phenomenological principles. It was constructed for an individual referred to here as Carl. Carl was a married man in his early middle-ages who worked as a teacher. He was suffering from insomnia, characterized by early morning awakenings, daily feelings of stress and tiredness, and low mood. He had been in cognitive-behavior therapy with mixed results; in addition, he had some experience with meditation. Experimental phenomenology was used to develop a morning meditation program (here referred to as a mindful embodiment) adapted to Carl's experiences and preferences. It was constructed in close collaboration with Carl. The 3-month treatment involved a combination of inperson sessions and email correspondence, with a 5-month follow-up. Although Carl wrote extensively about his experiences during this time, he was not motivated to provide quantitative ratings of his experiences or fill out psychometric questionnaires. The results are therefore described at a purely qualitative level. 


\title{
2.1 Methods
}

\subsubsection{Choice of Tense Points}

We used Jacobson's [17] progressive relaxation as a diagnostic tool to identify parts of the body where Carl felt especially tense. In Jacobson's [17] progressive relaxation, an individual is instructed to focus sequentially on each part of the body while tensing the muscles in each area of the body before relaxing them. The individual is asked to mindfully observe the difference between tension and relaxation throughout the exercise. On the basis of Carl's experiences during this exercise, four "tense spots" were selected that he should focus his attention on during the mindful embodiment practice: the eyes, mouth, neck, and chest. The focus on "the eyes" also included the area around the eyes. As to the mouth, Carl decided to focus specifically on his lips as he found that this was easier than focusing on the entire mouth and cheek region. He decided to focus on the neck following an experience during progressive relaxation when he felt a particular sense of relief as he pressed his neck back against the chair and then relaxed while noticing the difference between tension and relaxation.

Finally, the focus on the chest was natural because it was one of Carl's most tense areas. He reported feeling a "knot" in his chest, which was historically connected with a series of panic attacks over the years when he had felt sharp pain and tension in the chest and fears of an impending heart attack. This had led to several visits to emergency units at hospitals, where ECG was performed without any findings of physical problems, and benzodiazepines were prescribed. Although this had never led to the development of any full-blown panic syndrome, the tension in his chest was associated with considerable uneasiness.

\subsubsection{Development of a Personalized Meditation: Mindful Embodiment}

After a discussion of various possibilities, it was decided that when Carl had his early morning awakenings, he should stay in bed and find a suitable position for meditation. Carl decided to start from a position where he lay on his back, with one hand on the chest and the other on the belly. However, he also checked several other positions and alternated between these according to what felt best at the moment.

The meditation program was developed successively in collaboration with Carl and tested out by him during morning hours. The program was designed to express an explorative attitude toward the body and involve breathing as a central component. After experimenting with several possible selfinstructions, it was decided on Carl's suggestion to provide the self-instructions in a poetic form. The basic self-instructions, as described below, was applied first to the eyes, and subsequently in a similar manner to the lips, neck, and chest in that order.

\author{
May I explore \\ the feelings in \\ my eyes \\ by breathing into \\ that part of my body \\ slowly
}


This self-instruction was to be given silently with conscious attention to each line. The words were carefully selected. The phrase "May I explore" was selected to express an explorative attitude characterized by open receptiveness and curiosity, without excess focus on deliberate action, whereas the expression "the feelings in" defined the aspect of experience that was to be focused on, and "my eyes [lips, neck, chest]" defined the body region of immediate interest. The line "by breathing into" was designed to set a conscious focus on breathing. The following words, "that part of my body," were intended to facilitate the perception of the eyes (lips, neck, chest) as an integrated part of the body. The final word "slowly" was important because performing it slowly was assumed to increase the number of potential aspects of experience that were available for conscious attention.

Carl was instructed to start from the top (i.e., from the eyes) and work downward and repeat each self-instruction with a focus on each line. After going through the four tense spots, he was instructed to continue more spontaneously by shifting from one tense spot to another according to his feelings at each moment. Here, the long self-instruction could be substituted by a sequence of "short forms", such as (1) "Explore eyes (lips, neck, chest)"; (2) "Breathe eyes (lips, neck, chest)"; and (3) "Slowly eyes (lips, neck, chest)". Or in an even shorter version, the words "eyes", "lips", "neck", "chest," "explore", "breathe," and "slowly" could be repeated as such. When Carl got distracted from the exercise or got lost in thoughts, he was instructed to gently go back again to explore his eyes, eventually with the original long self-instruction and work downward again.

An additional part was the introduction of chords that combined two or more tense points. For example, the self-instructions could be "eyes and lips" or "neck and chest." Here, Carl was free to compose chords according to his spontaneous preferences at each moment, leading to a degree of improvisation and playfulness during the process.

When Carl was familiar with the mindful embodiment practice, he was encouraged to shift freely between these different components, leaving room for improvisation and playfulness within the given frame of the practice. In addition, Carl was encouraged to apply the same process to any other body part identified as tense. Figure 1 depicts the overall frame of the practice, with different bodily foci of attention as columns and attitude as rows.

\begin{tabular}{|l|l|l|l|l|l|}
\hline \multicolumn{5}{|c|}{ Focus of attention } \\
\cline { 2 - 6 } Attitude & Eyes & & & & \\
\hline Explore & & & & & \\
\hline Breathe & & & & & \\
\hline Slowly & & & & & \\
\hline
\end{tabular}

Figure 1 Basic components of the mindful embodiment practice. 


\subsubsection{Written Diary}

Although no quantitative measures were used, Carl was asked to maintain a diary and write down his observations regarding the variations in his practice of the meditation, as well as variations in his ensuing experiences. Moreover, he was encouraged to use Figure 1 to organize his observations. The contents of the diary were analyzed by thematic analysis [18].

\subsubsection{Ethical Considerations}

Informed consent was obtained from Carl, who read the manuscript and approved of publication. To preserve confidentiality, some personal details have been changed.

\subsection{Results}

Below is a summary of some of Carl's diary observations, formulated in terms of four themes. Most of these refer to immediate experiences during the mindful embodiment practice (referred to below as "the meditation", or "the practice"). At the end of the section, the results from a 5-month follow-up are summarized.

Positive short-term effects. Carl reported that he often liked to engage in the meditation during the morning. He described positive short-term effects in the form of increased relaxation and new aspects of body awareness. Sometimes, he fell asleep during the process but described this as a win-win situation. Falling asleep during the practice was not experienced as a failure because one of his problems was being unable to fall asleep after waking up early in the morning. And staying awake and engaging in the meditation was experienced as positive even if he would lie awake for a considerable time.

Occasional difficulties. The effects were not always positive. Sometimes, Carl could not concentrate on the task and was lost in thoughts, while being unable to fall asleep. Still, even on such unfocused occasions, he often enjoyed using one part of the practice: focusing on the neck while pressing the neck down against the bed and taking a deep breath. However, there were occasions (which were described as "exceptions") when he was distracted by thoughts to such an extent that he felt no positive effects at all. On these occasions, he often decided to rise from bed and stay up for a while (in accordance with instructions learned from his previous experiences with cognitive behavior therapy).

Transfer to other contexts. Carl tested using the meditation in other contexts. For example, although he seldom had problems falling asleep at night, he started to practice the meditation after having gone to bed in the evening. Previously, he had a habit of reading in bed until he felt sleepy; however, he now often preferred to lie down and practice the meditation because it made him feel relaxed and good about himself. The meditation was also transferred to moments of resting during the daytime. Sometimes, when taking a break, Carl would lie down for a while and spontaneously engage in the meditation. He reported experiences of relaxation and increased feelings of mental clarity as a result. In addition, he practiced it in situations that he usually experienced as "boring," such as waiting for the bus or sitting in a waiting room, and noted that the situation was no longer boring.

Mixed success in stressful situations. Carl tried using the meditation in some acute situations of stress, for example, in connection with a conflict at work. He reported a "mixed success" with this. 
Although he applied the self-instructions repeatedly and felt an effect in the form of at least a temporarily increased calm and relaxation, he lost concentration repeatedly due to intrusive thoughts. Still, he reported that it helped him make the feelings "less unbearable." Unfortunately, there were no quantitative data on the relative number of times a mixed-success was experienced, although Carl reported these occasional difficulties as "exceptions."

Managing chest pain. Carl had a history of occasional visits to medical emergency units due to chest pain with fears of an impending heart attack. Because no medical investigation showed any physical findings that could explain the pain, Carl finally concluded that this type of chest pain was nothing to be afraid of. Now, he reported an occasion where he had felt the well-known pain in the middle of the chest and managed it by lying down and using the self-instruction in the mindfulness embodiment practice, with a focus on the chest: "May I explore the feelings in my chest by breathing into that part of the body, slowly," followed by repeating the short form "Explore chest," "Breathe chest," "Slowly chest." The chest pain receded within minutes, and although it returned a while later, he managed it in the same manner with a similar effect.

Five-month follow-up. Five months after the end of the treatment, Carl was contacted and asked to report his experiences from practicing the meditation. Again, no quantitative measures were recorded; however, Carl reported that he had continued the practice and he enjoyed it. Early morning awakenings were no longer reported to be a problem. Most conspicuously, he reported that the practice helped him when he had experienced pain and tension in his chest (he guessed that this had occurred around ten times). Typically, he had used the entire mindful embodiment practice on these occasions; that is, he had not only focused on the chest but also included the eyes, lips, and neck, and used the self-instruction to "explore," "breathe into," and "go slowly." He described an experience of how this "helped to untie a knot in his chest", as if each body part represented a "thread in the knot." He described a series of successful experiences of managing his chest pain, with one exception. Usually, the mindful embodiment practice had an almost instantaneous effect, but on one occasion it did not work as expected. Carl attributed the lack of effect at that time to his "slipping into an impatient, goal-directed attitude" to achieve an effect as fast as possible in an instrumental manner. He reflected that it might be essential to hold a nongoal-directed attitude, focused on "being rather than doing" while practicing this exercise.

\section{Discussion}

The present study illustrates the principles of experimental phenomenology. However, it does in no way aspire to present a generalized treatment for insomnia. The mindful embodiment practice was a personalized health intervention developed for a specific individual in close collaboration with that person. The purpose of the study was to illustrate how a personalized health intervention can be developed using the principles of experimental phenomenology.

Personalized health interventions need to be adapted to the client's preferences and previous knowledge and skills. In this case, the individual had previous experience with both cognitive behavior therapy and meditation. For a client without this kind of experience, an optimal program would probably have to be different. In addition, self-instructions were formulated in the form of a poem on Carl's suggestion. Although the poetic form suited his preferences very well, it could be less appropriate with another client. 
Although experimental phenomenology is primarily a person-oriented form of research, it also aspires to generate generalizable knowledge. However, the basic principle is to start from the individual case and search for similarities and differences across cases. Generalized conclusions may be possible at several levels. Generalizations at a lower level of abstraction refer to specific phenomenological practices and how they are to be conducted most efficiently. One question is to what extent it is possible to formulate general conclusions at this level, which take the role of the context and the individual person into account. At a higher level of abstraction, we may also ask if it is possible to formulate general conclusions that can be applied to all phenomenological practices. A candidate for such a high-level generalization would be that proceeding slowly is generally more effective. But is it always more effective, in all contexts, for all individuals, and independently of the kind of phenomenological practice? And is there a linear association between tempo and impact, or is the relationship of a curvilinear nature so that there is an optimal tempo, with a loss of effect when one proceeds too slowly? Another possible hypothesis of a generalized nature would be that a non-goal-directed attitude (an abstention from willful efforts), focused on being rather than doing, may be essential to the effects of these kinds of practices.

Although such more theoretical questions are of definite interest to experimental phenomenology, the primary ambition here was to develop and document phenomenological practices at the level of the individual person and to test their effects at this person-specific level. Before this is conducted to a sufficient degree, it may be premature to try to formulate more general conclusions. In this research, it may be important to measure relevant variables not only before and after treatment but also during the treatment to obtain data on variations during the treatment process that can provide potential information about processes involved and change mechanisms (cf. [19]).

One major limitation of the present case illustration was the absence of quantitative data, which could have made it possible to compare the quality of sleep and other relevant characteristics before and after the treatment. Although Carl was highly motivated to engage in this kind of treatment, as seen both in his active collaboration during all parts of the process and in his extensive writings about his experiences, he was not equally motivated to make quantitative ratings of his experiences. Future health interventions based on experimental phenomenology may benefit by including quantitative measures not only before and after treatment but also during the process to substantiate the nature of variation and change and the progress during the treatment.

\section{Author Contributions}

LGL wrote the article.

\section{Competing Interests}

The author has declared that no competing interests exist.

\section{References}

1. Lundh LG. Experimental phenomenology in mindfulness research. Mindfulness. 2020; 11: 493506. 
2. Lundh LG. Experimental phenomenology as an approach to the study of contemplative practices. Front Psychol. 2022; 12: 751298.

3. Husserl E. The crisis of the European sciences and transcendental phenomenology. Evanston, IL: Northwestern University Press; 1970.

4. Ihde D. Experimental phenomenology. An introduction. New York: G.P.Putnam's Sons; 1977.

5. Ihde D. Experimental phenomenology. 2nd ed. New York: State University of New York; 2012.

6. Thompson E. Why I am not a Buddhist. London: Yale University Press; 2020.

7. Kabat-Zinn J. Full catastrophe living: Using the wisdom of your body and mind to face stress, pain and illness. New York: Bantam Books; 2013.

8. Shapiro SL, Carlson LE. The art and science of mindfulness: Integrating mindfulness into psychology and the helping professions. Washington D.C.: American Psychological Association; 2017.

9. Langer EJ. Mindfulness. Paris: Hachette Books; 2014.

10. Bergman LR, Lundh LG. Introduction: The person-oriented approach: Roots and roads to the future. J Pers Oriented Res. 2015; 1: 1-6.

11. Lundh LG. The person as a focus for research - The contributions of Windelband, Stern, Allport, Lamiell and Magnusson. J Pers Oriented Res. 2015; 1:15-33.

12. Espie CA. Insomnia: Conceptual issues in the development, persistence, and treatment of sleep disorder in adults. Annu Rev Psychol. 2002; 53: 215-243.

13. Ong JC, Ulmer CS, Manber R. Improving sleep with mindfulness and acceptance: A metacognitive model of insomnia. Behav Res Ther. 2012; 50: 651-660.

14. Lundh LG, Broman JE. Insomnia as an interaction between sleep-interfering and sleepinterpreting processes. J Psychosom Res. 2000; 49: 299-310.

15. Lundh LG. The role of acceptance and mindfulness in the treatment of insomnia. J Cogn Psychother. 2005; 19: 29-39.

16. Lundh LG. Insomnia. In: Mindfulness and acceptance in behavioral medicine: Current theory and practice. Oakland: New Harbinger Publications; 2011. pp.131-158

17. Jacobson E. Progressive relaxation. Chicago: University of Chicago Press; 1938.

18. Braun V, Clarke V. Using thematic analysis in psychology. Qual Res in Psychol. 2006; 3: 77-101.

19. Lundh LG, Falkenström F. Towards a person-oriented approach to psychotherapy research. J Pers Oriented Res. 2019; 5: 65-79. 
OBM Integrative and Complementary Medicine 2022; 7(1), doi:10.21926/obm.icm.2201008

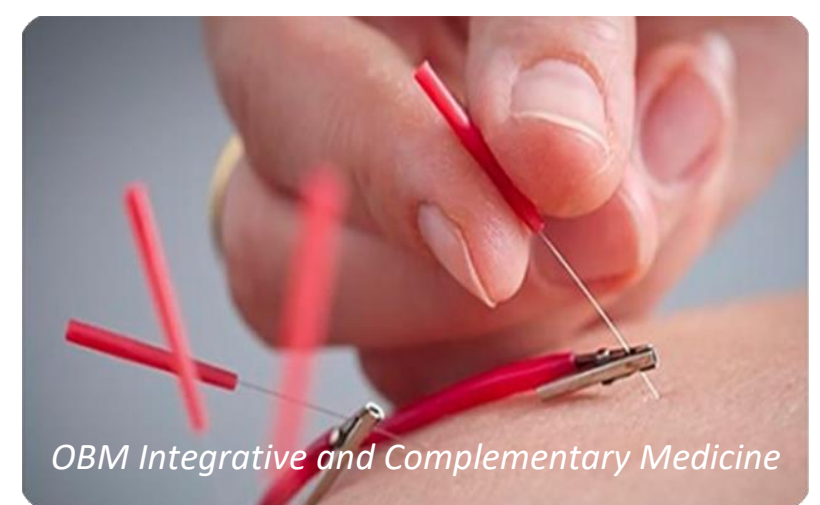

Enjoy OBM Integrative and Complementary Medicine by:
1. Submitting a manuscript
2. Joining in volunteer reviewer bank
3. Joining Editorial Board
4. Guest editing a special issue
For more details, please visit:
http://www.lidsen.com/journals/icm 